\title{
Intercambios sobre la noción de paisaje cultua: la experiencia en la ciudad de Daxu [Jianghua County, Hunan] China
}

\author{
SELECCIÓN VIII SEMINARIO INTERNACIONAL DE INVESTIGACIÓN EN URBANISMO
}

\author{
Paula Orduña \\ Lic. en Geografía y MSc. en Urbanismo. Universitat Politècnica de Catalunya. Barcelona [Cataluña] España. \\ $<$ paula.orduna@upc.edu>

\section{Melisa Pesoa} \\ Arquitecta y Dra. en Urbanismo. Universitat Politècnica de Catalunya. Barcelona [Cataluña] España. \\ $<$ melisa.pesoa@upc.edu>

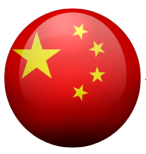

\section{Huan Wu} \\ Arquitecto, por la School of Art and Architecture of the Central South University of Changsha (CSU); y MSc. \\ en Urbanismo en la Universitat Politècnica de Catalunya. Barcelona [Cataluña] España. \\ <wuhuan.cn@foxmail.com>
}

\section{Resumen}

El presente artículo trata de condensar la experiencia fruto del intercambio académico de ideas sobre la incorporación de la noción de "paisaje cultural" como instrumento de planeamiento urbano y territorial en un pequeño pueblo de tradición rural del interior de China llamado Daxu. En el contexto del acelerado proceso de transformación urbana y territorial propio del gran país asiático, se afronta la problemática de incorporar el concepto de "paisaje cultural" como eje de las actuaciones del máster plan de este municipio rural, con el objetivo de ayudar a un desarrollo local sostenible.

\section{Palabras clave}

Paisaje cultural. Intercambio académico. Planeamiento urbano y territorial. China.

\section{Exchanges on the notion of cultural landscape: the experience in the city of Daxu [Jianghua County, Hunan] China}

\begin{abstract}
This article tries to summarize the experience of an academic exchange on the incorporation of "cultural landscape" notion as an instrument for urban and regional planning in a small Chinese village called Daxu. In the context of Chinese's accelerated urban and territorial transformation process, this experience tackles the problem of incorporating the concept of "cultural landscape" in this rural municipality master plan in order to create a sustainable local development.
\end{abstract}

\section{Keywords}

Cultural landscape. Academic exchange. Urban and Territorial planning. China.

\section{摘要}

“文化景观”成为一种城市和区域规划方法。本文描述了通过国际学术合作的经验，尝试把这种 方法应用于中国腹地的一个小型传统村落的城市和区域发展规划。中国高速发展的城市和区域 转型背景下，在乡镇总体规划中引入“文化景观”的概念可以作为解决城乡发展问题的工具。 


\section{Introducción}

Este artículo resume la experiencia de un intercambio académico realizado entre 2014 y 2015 entre la School of Art and Architecture of the Central South University of Changsha (CSU) y el Departamento de Urbanismo y Ordenación del Territorio de la Universitat Politècnica de Catalunya (UPC) de Barcelona.

La colaboración entre universidades y el municipio de Daxu se enfoca no desde la perspectiva de una transferencia de conocimientos, sino como un proceso de aprendizaje recíproco a través de grupos de trabajo paralelos, encargados de definir líneas de investigación y proyecto para enriquecer tanto el proceso pedagógico universitario como, también, el proyecto real de ensanche de una pequeña ciudad en el Sur de China. Con este intercambio se busca aunar planteamientos y conceptos del planeamiento urbano y territorial fundamentados en la puesta en valor de los recursos locales endógenos, tanto naturales como paisajísticos y culturales. El objetivo que se plantea es que un municipio relativamente pequeño que quiere crecer lo pueda hacer de una manera alternativa a la planteada desde la administración, reajustando el plan de renovación y extensión urbana existente.

Los ejes de trabajo incluyen diferentes temáticas a diferentes escalas:

- desarrollo socio económico y ensanche de la ciudad

- refuerzo y puesta en valor de la identidad cultural de la minoría Yao

- preservación del patrimonio cultural y arquitectónico

- preservación del patrimonio natural de origen kárstico y valorización del uso agrícola

\section{Un pequeño pueblo en crecimiento}

Daxu (Daxuzhen, 大圩) es un municipio de 30.000 habitantes situado en un pequeño valle rodeado de cordilleras de relieve kárstico en el extremo Sur de la provincia de Hunan (Figura 1). La provincia de Hunan se ha convertido en los últimos años en un importante zona de producción siderúrgica, maquinaria y electrónica, a medida que la industria ha ido expandiéndose desde las provincias costeras (Guangdong y Zhejiang) hacia el interior. La ciudad prefectura de Yongzhou, que integra el condado autónomo de Jianghua — donde se ubica Daxu一, linda con las provincias de Guangdong (Cantón), una de las provincias más ricas y pobladas de China, y Guangxi, menos dinámica económicamente y limítrofe con Vietnam.
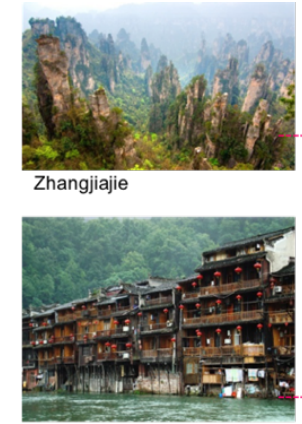

Fenghuang Ancient Town

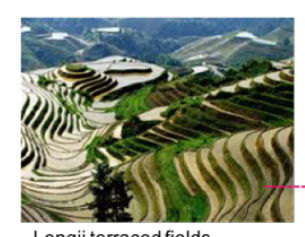

Longji terraced fields

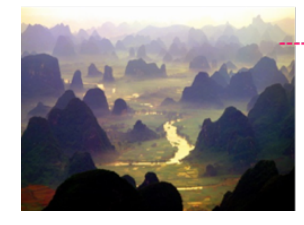

Húnán province 湖南省

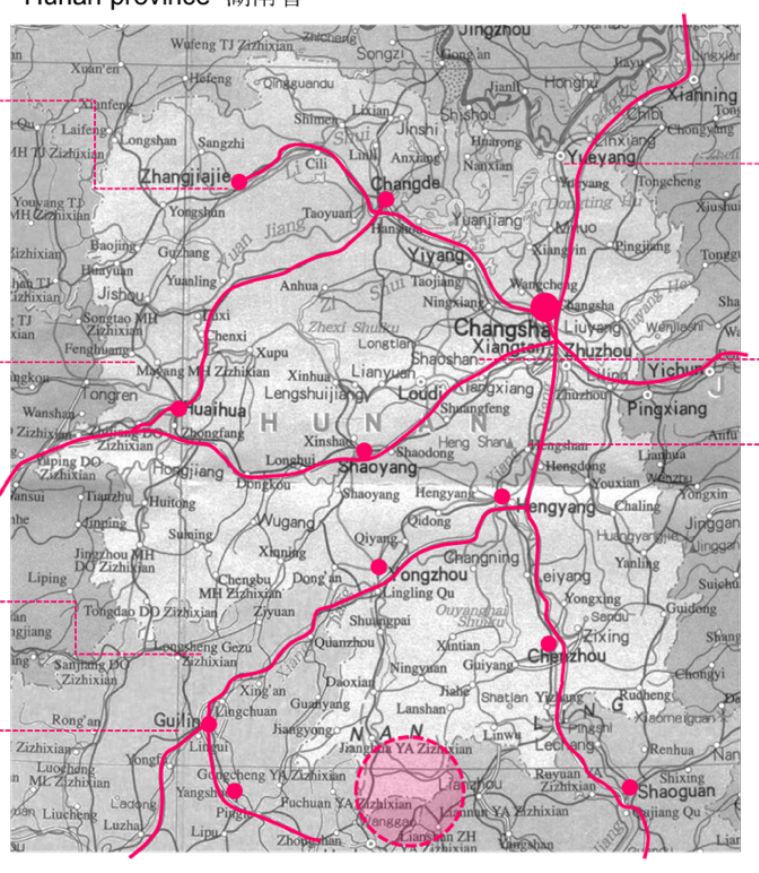

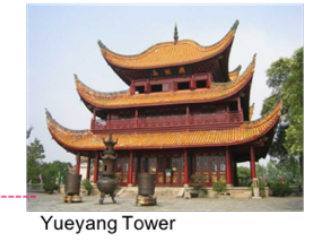
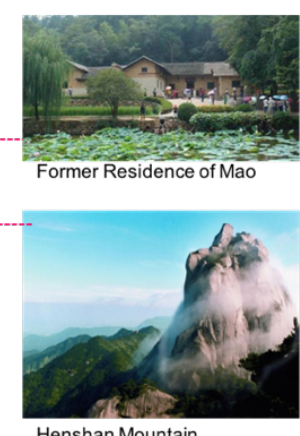

Henshan Mountain

Figura 1. Localización del municipio de estudio en círculo, Daxu Town, Jinghua County, Hunan province (China). Se observan también los diferentes sitios de interés cultural y natural de la provincia de Hunan y Guangxi. Fuente: Elaboración propia. 
La orografía ha aislado históricamente el valle cerrado por montañas en el que se ubica Daxu, que no ha participado de las dinámicas territoriales de desarrollo económico e industrial de otras áreas próximas. Una de las principales consecuencias de este aislamiento ha sido el éxodo de la población local en edad de trabajar hacia áreas más industrializadas, en detrimento de la actividad rural local, dejando en el municipio una mayoría de ancianos y niños.

El condado autónomo de Jianghua se caracteriza por la elevada concentración de población perteneciente a la minoría étnica Yao. Según Wiens (1987), la minoría Yao, originaria de Hunan, se retiró con la conquista de territorio de la etnia y dinastía Han (la etnia mayoritaria en China) hacia las montañas entre Hunan, Guizhou Cantón y Guangxi, extendiéndose hasta el Este de Yunnan hacia el año 100 a.C.

En la actualidad, el crecimiento urbano del municipio de Daxu viene determinado por la mejora de las infraestructuras de comunicación que conectan este territorio otrora aislado con algunas ciudades cercanas. Con un tren de alta velocidad es posible llegar desde Changsha hasta Chen Zhou, y de allí con un autocar, llegar a Daxu en unas 4 horas. A pesar de las mejoras, Daxu sigue teniendo una accesibilidad un tanto complicada, no obstante se acerca progresivamente a zonas más pobladas y se presentan nuevas posibilidades de desarrollo económico, ligadas tanto a las actividades comerciales como al turismo cultural.

Hasta hace poco, la economía de este territorio se basaba en una agricultura diversificada y la producción manufacturera de derivados agrícolas (como el tabaco, arroz o maíz). En los últimos decenios la base económica ha cambiado de la actividad agraria al comercio y los servicios. La difícil conectividad, que imposibilitó el desarrollo económico antaño, ha servido en contrapartida para preservar sus recursos naturales, agrícolas y culturales del acelerado proceso de urbanización general del gran país asiático (Figura 2).

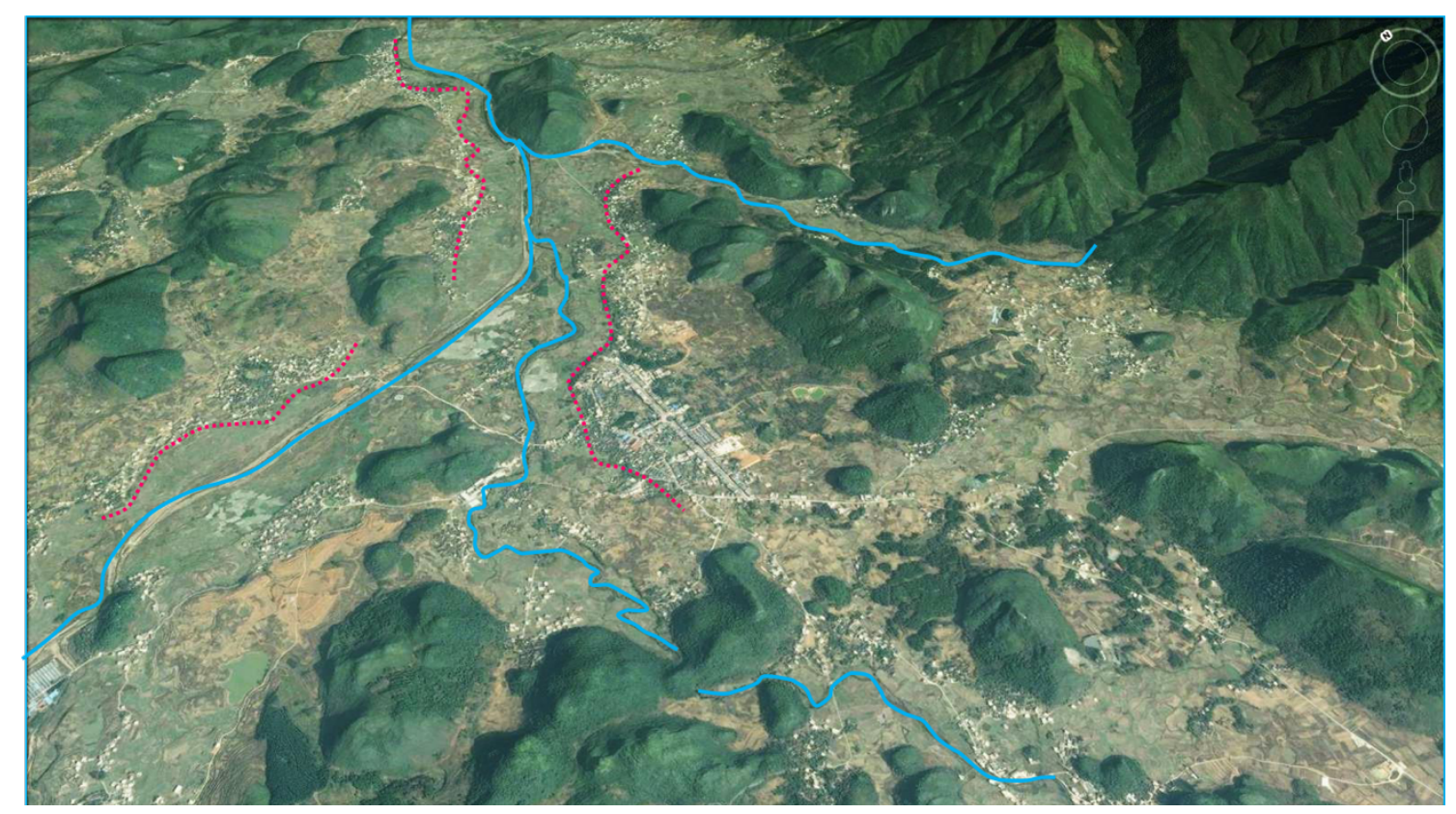

Figura 2. Daxu en contexto. Se observa la ciudad y su ensanche a partir de una avenida en el centro de la imagen, rodeado del paisaje kárstico, característico de la región. En azul se marcan los cursos de agua más importantes y en línea rosa de puntos los límites de las áreas inundables. Fuente: Elaboración propia sobre base de Google Earth.

Pero, por otro lado, la necesidad de plantear un ensanche para Daxu responde también a la urgencia de relocalizar cerca de 7.000 habitantes pertenecientes a 16 poblados que en viven en zona de riesgo ambiental. La mayor parte de esta población pertenece a la etnia Yao y ocupa actualmente una área más remota y montañosa al Sudoeste del valle de Daxu (Figura 4), llamada Liangchahe, que es considerada de alto riesgo ambiental y susceptible de padecer deslizamientos, en buena medida originados por la entrada en funcionamiento de una presa que ha alterado las dinámicas geomorfológicas de la región. 


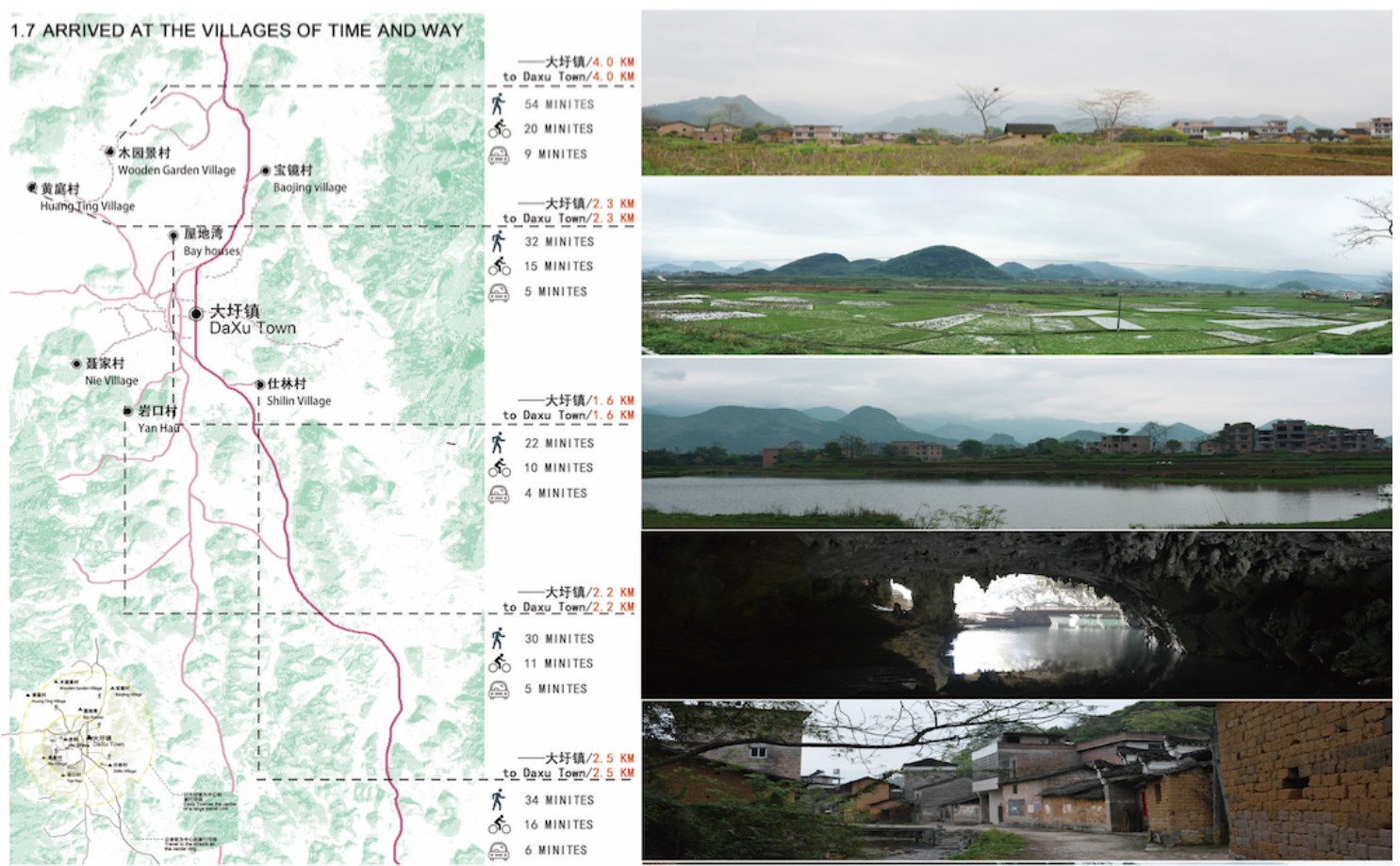

Figura 3. Distancias a otros núcleos cercanos y paisajes característicos de la zona. Fuente: Dibujos y fotografías de los alumnos Wenbo Zhang, An Dai, Yunbo Deng, Yajun Liu, Jiexin Liao.

Además de los riesgos ambientales, esta población enfrenta actualmente otras problemáticas. Por un lado, la mayoría de población permanente son niños y mujeres ancianas, que carecen de servicios básicos, por lo que la condición de aislamiento hace que sea muy difícil llevar ayuda cuando se necesita. Además, la economía local se encuentra enormemente desfavorecida: la erosión está provocando la pérdida de minerales y materia orgánica de la capa superficial del suelo, lo que reduce su fertilidad. Ello imposibilita una producción de recursos suficiente y los habitantes se ven obligados a bajar constantemente a comprar alimentos en Daxu.
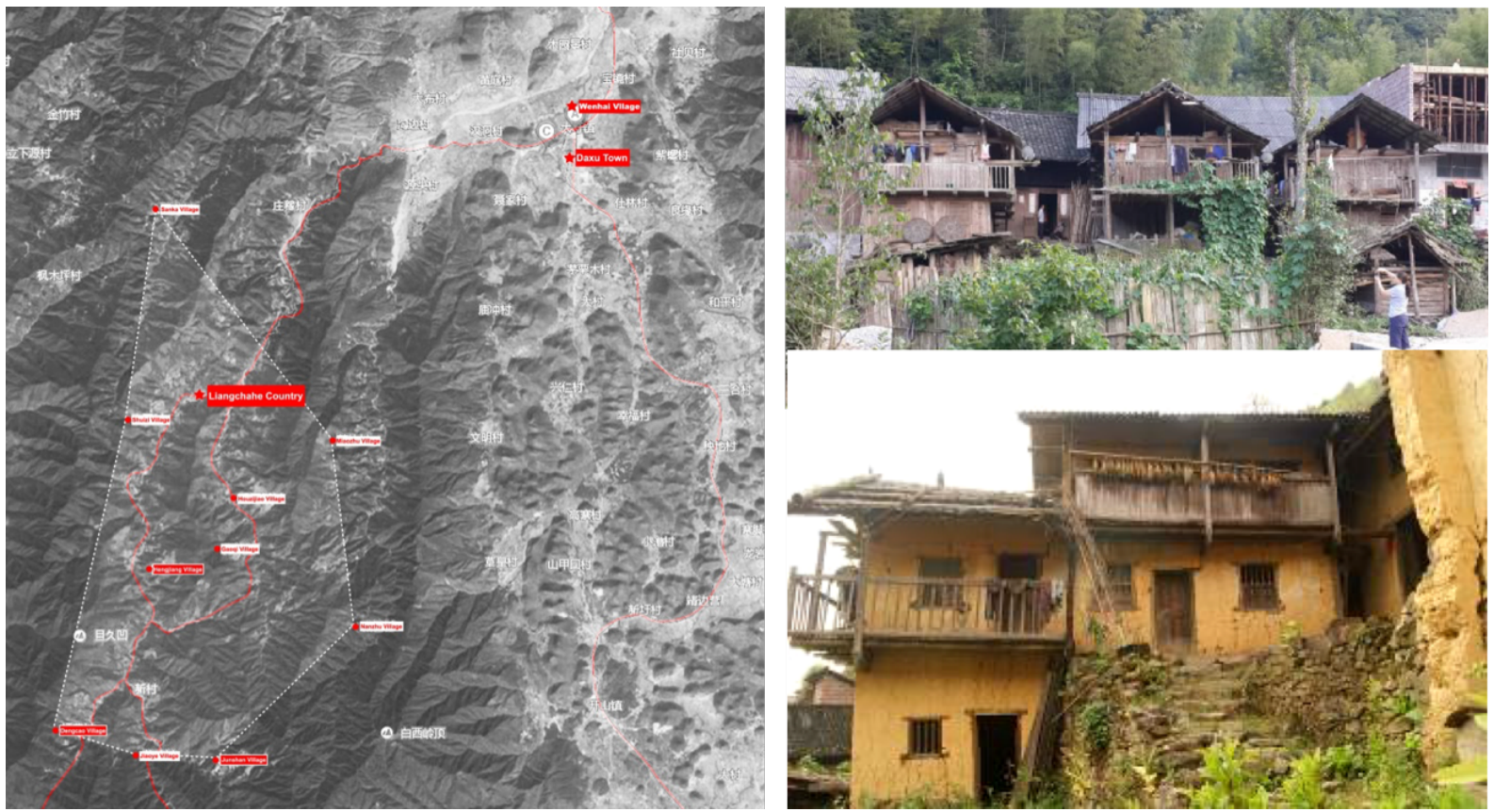

Figura 4. Área donde se ubican las poblaciones de montaña a relocalizar y su relación respecto a Daxu. También se muestra el área donde se piensa realojar a estas familias, un sector al Norte de Daxu, llamado Wehai. A la derecha, una muestra de la arquitectura típica de las comunidades a realojar. Fuente: Elaboración propia. 
La idea del municipio es relocalizar a los habitantes de las montañas en una zona rural al Norte de Daxu, llamada Wenhai Village. Aquí habitan también miembros de la etnia Yao, pero con hábitos y costumbres diferentes, propias de la llanura.

Adicionalmente a esta situación, se presenta el caso singular de un antiguo poblador de Daxu, que tras hacer una buena fortuna comercial en Guangdong, desea reinvertir parte de su dinero en un emprendimiento vinculado al arte y a la valoración del paisaje de su ciudad natal, con un equipamiento que quizá atraiga a artistas de todo el mundo a este paisaje singular.

El intercambio académico se divide en dos grandes etapas. La primera, busca planificar el desplazamiento y relocalización de la nueva población; en este sentido, la tarea es proponer un reajuste al máster plan (previsto para 2013-2030) planteado por el municipio integrando los recursos paisajísticos del territorio. En segundo lugar, se plantea la comprensión de referencias al patrimonio y la identidad cultural de la etnia Yao como recurso para el desarrollo local y la posible llegada de visitantes al área a través del desarrollo de un turismo especializado. Desde el Departamento de Urbanismo y Ordenación del Territorio de la Universitat Politècnica de Catalunya, la directriz principal del trabajo fue incorporar el concepto de "paisaje cultural" como forma de interpretar las características y preexistencias naturales, agrícolas, culturales y paisajísticas del territorio para poder proyectar sobre éste. Antes de entrar en detalle sobre el trabajo propiamente dicho, presentaremos algunas características de la población local y sus activos culturales.

\section{El "paisaje cultural", un concepto para proyectar alternativas}

El máster plan que propone el municipio de Daxu determina un crecimiento guiado por una malla abstracta que no tiene en cuenta las particularidades del territorio y destruye una zona de producción agrícola importante. La idea es introducir el concepto de "paisaje cultural" en el proceso pedagógico y en el proyecto, para repensar el planeamiento urbano y territorial local y considerar cuál puede ser su aplicación en el contexto chino.

El primero en acuñar el concepto de "paisaje cultural" fue el geógrafo americano Carl Sauer (1889-1975), fundador de la Escuela de Berkeley. Fue también el promotor de una nueva corriente de pensamiento geográfico que, alejándose de la aproximación corológica a la superficie terrestre propia de la geografía regional francesa, se interesa por la morfología de los paisajes culturales. La cultura - material o inmaterial- posibilita que los seres humanos modifiquemos el paisaje natural, lo que desemboca en la producción de un "paisaje cultural" determinado (Sauer, 1925).

Los geógrafos culturales Wagner y Mikesell (1962), puntualizan que esta interrelación entre potenciales culturales de una población determinada y el conjunto natural del territorio que ocupan, es el resultado de una larga evolución tanto natural como de esfuerzos antrópicos:

For [...] cultural geographers, any sign of human action in a landscape implies a culture, recalls a history, and demands an ecological interpretation. [...] The history of any people evokes its setting in a landscape, its ecological problems, and its cultural concomitants; and the recognition of a culture calls for the discovery of traces it has left on the earth (Wagner \& Mikesell, 1962, p. 23).

En 1992 el Comité de Patrimonio Mundial de UNESCO incluye la categoría de "paisaje cultural" en sus Directrices Prácticas. El concepto se define como "obra conjunta entre el ser humano y la naturaleza" (Conti, 2012). Esta categoría evidencia procedimientos e instrumentos de planeamiento sostenibles pues consideran las características y limitaciones de los entornos naturales en los que se despliegan.

La preocupación por el planeamiento urbanístico y territorial ha evolucionado notablemente y en el siglo XXI se avanza hacia un nuevo paradigma de sustentabilidad territorial.

Tal como apunta Sabaté (2015), los planes y proyectos de mayor interés y carácter innovador priorizan ahora un nuevo binomio: naturaleza y cultura; naturaleza y cultura como partes de un 
mismo concepto: patrimonio. En este contexto, la categoría de "paisajes culturales" puede convertirse en un instrumento de planeamiento de gran relevancia pues "constituyen la expresión de la memoria, de la identidad de un territorio, que se puede ir enriqueciendo sucesivamente" (Sabaté, 2015).

El concepto de "paisaje cultural" cobra aún más relevancia en el contexto de urbanización acelerada de China. La lectura e interpretación del territorio puede contribuir a proyectar el futuro crecimiento en el caso de Daxu. Este esfuerzo de "lectura intencionada" es la base del instrumento que proponemos y constituye un método para construir entornos más diversos y cargados de identidad que a la vez den alternativas de desarrollo económico.

Con la noción de "paisaje cultural" pretendemos aproximarnos a la renovación y el enriquecimiento del enfoque del actual máster plan de Daxu. A partir de la comprensión de los atributos particulares del territorio, como son la identidad cultural Yao, el paisaje kárstico y la tradición agrícola local se pueden reformular los criterios de ordenación, o, dicho de otra manera, formular acciones más ajustadas a las necesidades específicas de este territorio, incorporando propuestas de disciplinas que estudian la naturaleza, el paisaje, la cultura y las identidades locales.

\section{Activos culturales de Daxu y alrededores}

En primer lugar, y en un trabajo conjunto entre alumnos y profesores de ambas universidades, elaboramos una primera clasificación de los recursos disponibles, dividida en dos grandes grupos, naturales y culturales, siguiendo la metodología planteada por Sabaté y Schuster (2001) en el proyecto del Eje Llobregat. Siempre teniendo en cuenta que un recurso nos permitirá explicar una historia, construir un relato que sirva no sólo para proyectar sino para que la población tenga presente su historia y su aporte y lo valorice y proteja.

Tabla 1. Recursos naturales y culturales de Daxu y alrededores. Fuente: Elaboración propia.

\begin{tabular}{|l|l|}
\hline 1. Naturales & $\begin{array}{l}\text { Ríos } \\
\text { Colinas (relieve kárstico) } \\
\text { Bosques }\end{array}$ \\
\hline 2. Culturales & $\begin{array}{l}\text { Casco antiguo del pueblo } \\
\text { Arrozales } \\
\text { Cultivos de secano } \\
\text { Aspectos intangibles de la etnia Yao } \\
\text { Antigua Villa Baojing (s.XVII) }\end{array}$ \\
\hline
\end{tabular}

\subsection{Recursos naturales: Relieve kárstico y tradición agrícola}

El área de estudio está drenada por los afluentes del río Tuojiang. Se trata de una área montañosa con gran variedad climática y paisajística. El clima es subtropical y húmedo y las lluvias quedan concentradas distinguiéndose una época seca de una húmeda. El Suroeste de China - concretamente las provincias de Guangxi, Guizhou, Yunnan, Sichuan, Hunan, Hubei- presenta los relieves kársticos más grandes y variados del mundo (Botazzi et al. 2011). Se trata de formaciones kársticas tropicales y subtropicales de áreas montañosas húmedas y de frondosa vegetación que oscilan entre los 700 y los $5.600 \mathrm{~m}$ de altitud. Algunos de estos sitios han recibido el estatuto y reconocimiento de Patrimonio mundial de la UNESCO, como en el caso del área paisajística de Wulingyuan, al Norte de la misma provincia de Hunan.

El área de estudio conforma una combinación de gran interés paisajístico entre el modelado kárstico y la riqueza y tradición agrícola. Las formas kársticas rodean el asentamiento por el Este y el Sur. Se trata de mogotes o pequeñas montañas muy escarpadas que restan de la topografía anterior a la erosión kárstica. El paisaje actual es el resultado de una "competición entre el levantamiento del Himalaya y la erosión de rocas calcáreas des de mediados del Terciario" (Botazzi et al., 2011). 
La agricultura de la zona de estudio es próspera gracias a la humedad de su clima tropical. Se trata de una de las áreas con mayor producción de arroz y té. Además existen otros productos agrícolas como el ruibarbo, el almizcle, la miel, el tabaco, el cáñamo y el ramio para elaborar fibras textiles.

La abundancia agrícola de este territorio se hace patente en Daxu. El municipio queda rodeado por una vasta área agrícola que limita a su vez con las montañas resultado de la erosión kárstica. Los alrededores de Daxu conforman un mosaico de diferentes cultivos donde destacan los arrozales que se combinan con áreas de bosque disperso de pino, arce y eucaliptus. Dentro de la trama urbana consolidada coexisten huertas y otros cultivos de secano de subsistencia. El objetivo es posibilitar mediante el planeamiento que este mosaico perdure y pueda ser integrado en el futuro desarrollo urbanístico.

\subsection{Recursos culturales: La minoría étnica Yao y la ciudad antigua}

China es un crisol de diferentes pueblos y culturas. Con el ascenso al poder del partido comunista chino en 1949, se inicia un amplio programa de censo etnológico en la República popular china que concluye con el reconocimiento de una etnia mayoritaria, la Han y de cincuenta y cinco etnias minoritarias.

Las distinciones étnicas responden ante todo a criterios lingüísticos, pero también hacen referencia a las diferentes tradiciones y cultos existentes. Cuantitativamente, el conjunto de las minorías étnicas suma en China hasta 114 millones de personas de un total de 1,3 mil millones de personas y ocupan hasta cinco octavas partes del territorio chino, concentrándose en el oeste del país.

A pesar de una Constitución que garantiza la igualdad de derechos entre todas las nacionalidades e impide la discriminación protegiendo los intereses legítimos como la libertad de utilizar su lengua, escritura y preservar sus costumbres, las reformas económicas y sociales iniciadas a partir de 1979 han creado fuertes disparidades sociales y económicas que las minorías étnicas padecen de manera particular.

En lo que respecta la minoría étnica Yao, la lenta colonización Han les obligó a ocupar lugares inhóspitos del sur de China y de países del Sudeste asiático. Actualmente, este grupo étnico habita zonas montañosas del Sur y Suroeste chinos extendiéndose a países limítrofes como Birmania, Laos y Vietnam. En 2003 su población en China se estimaba en 2,6 millones de personas ${ }^{1}$.

Esta etnia presenta características culturales muy particulares. Entre estas características destacan su vistosa vestimenta, marcada por un color azul intenso. Su cultura gastronómica tiene también elementos diferenciadores y entre los productos locales destacan el vino, la carne de cerdo, té, y el tofú. Entre los festivales destacan el Festival de fuegos artificiales, el festival del Pájaro, del noviazgo y la cosecha (Bird festival for courtship and harvest), la conmemoración de Pan King (que fue el primer líder Yao), en tanto que en el patrimonio cultural intangible podemos destacar la cultura Fete y el baile de percusión Long-drum (Figura 5).

Cabe destacar que en los últimos años en China las minorías étnicas reciben un tratamiento estrechamente vinculado al turismo que desde el punto de vista occidental puede llegar a resultar folclórico y museizante, pero es la forma en que ellos ponen en valor su cultura. No obstante, confiamos en que existen otras formas menos "literales" de poner en valor este valioso activo.

El casco antiguo de Daxu, en forma del almendra, constituye un importante recurso cultural, porque mantiene buena parte de la estructura y de la arquitectura tradicional, construida en madera, sobre todo en la parte Norte. En general, las construcciones son de tres plantas, donde la planta baja se destina a almacén, la planta primera a vivienda y la planta alta para la conserva del grano. En caso de inundación, los edificios también funcionan como refugio al estar la vivienda en altura.

Al Norte de Daxu, existe una pequeña Villa llamada Baojing, que constituye otro importante recurso cultural. Esta villa se compone de cinco grupos de edificios construidos en el siglo XVII. El mayor

\footnotetext{
${ }^{1}$ Ver: http://www.stats.gov.cn/english/statisticaldata/yearlydata/yarbook2003 e.pdf
} 
grupo de edificios, XinWu, está constituido por tres salas de familia, nueve patios, dieciocho salas privadas, un total de ciento ocho habitaciones. Estas habitaciones se organizan de forma muy regular, siguiendo los patrones espaciales y tecnológicos de la arquitectura tradicional china.

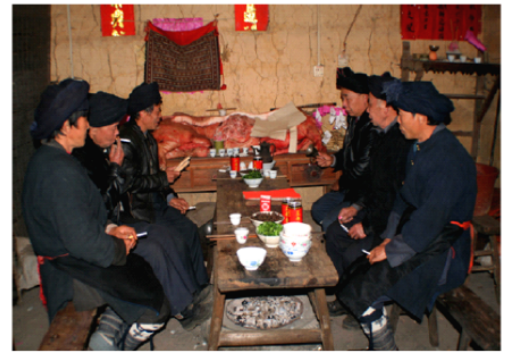

坐席读书 Reading

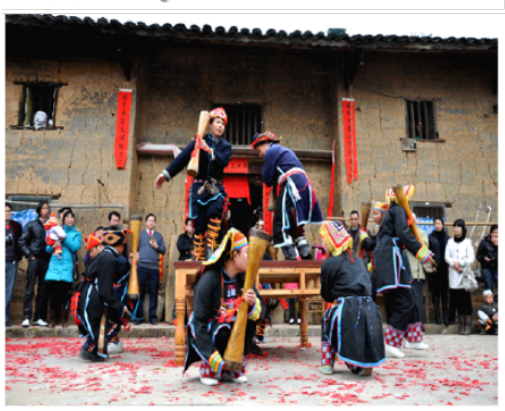

长鼓敬神 Worship to Gods

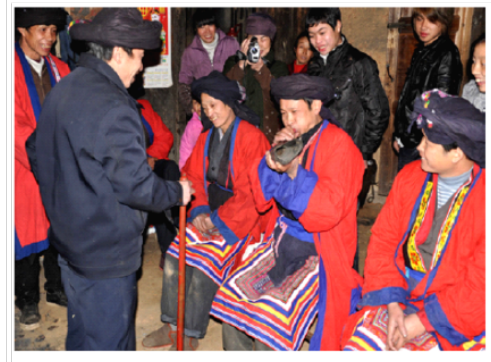

学 法 Yao rabbi

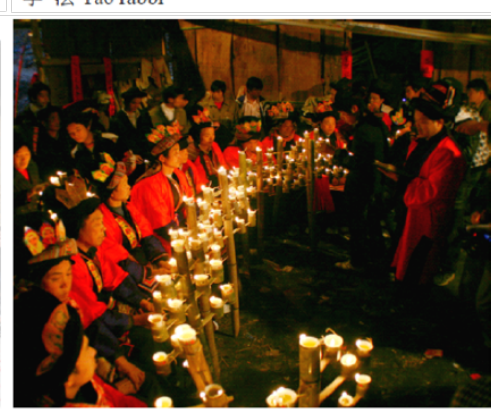

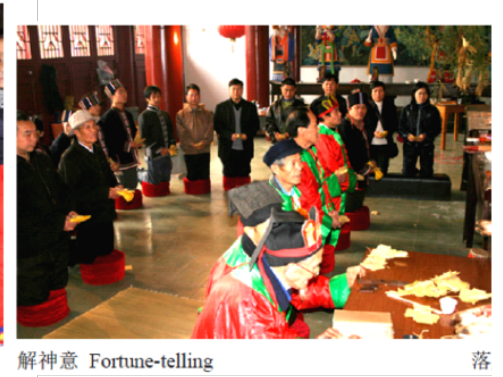

解神意 Fortune-telling

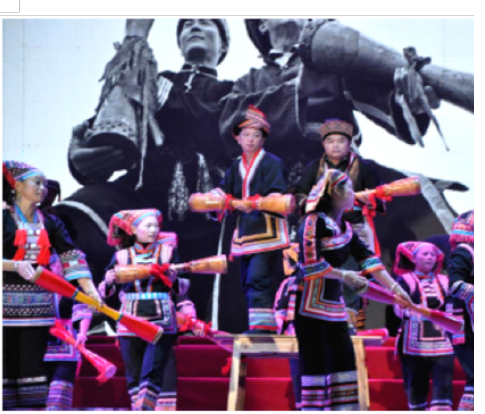

Figura 5. Algunas manifestaciones culturales de la etnia Yao. Fuente: Google Images.

Los edificios de Baojing son una muy buena muestra de la antigua vivienda china local y debido a que se encuentran lejos de una gran ciudad, mantienen una buena relación con el entorno rural y conservan su arquitectura original. Con algún tipo de rehabilitación, algunos edificios antiguos podrían volver a ser utilizados.

El problema general que enfrenta Daxu y sus alrededores, tiene que ver con que, debido a la insuficiencia de la producción, no se puede mantener una vida basada en la gran familia rural tradicional, y muchos jóvenes tienen que abandonar el pueblo en busca de mejores oportunidades en las ciudades de rápido desarrollo y la mayoría de ellos no vuelve a vivir al pueblo.

\section{Reconduciendo el master plan}

Hace 10 años, el municipio de Daxu se planteó crecer hacia el Este sobre la base de un eje rectilíneo en sentido Norte Sur, a modo de una gran avenida de $24 \mathrm{~m}$, que permitió crear nuevas parcelas de $18 \mathrm{~m}$ de ancho y edificar construcciones más o menos homogéneas que arman un frente continuo de planta baja +3 pisos superiores. Este eje descentró, como era de esperar, la actividad de la ciudad, cuyos principales comercios ahora se ubican sobre la avenida.

En el año 2013, el municipio propuso un segundo crecimiento, con el objetivo de ensanchar más la ciudad, generando nuevas parcelas edificables, con el añadido de la necesidad de relocalizar a la población desplazada de las montañas (Figura 6). Se planificó así un "ensanche" en malla, basado en la avenida, que conforma unas manzanas de grandes dimensiones, unos 250 x $250 \mathrm{~m}$. Éste crecimiento se extiende sobre terrenos hoy cultivados, planteando una continuidad vial con las calles perpendiculares al eje y acercándose poco a poco a la colina Este.

La CSU de Changsha junto con los profesionales del municipio, se proponen generar una alternativa al master plan, que involucre los valores culturales del lugar, más allá de la construcción de una simple malla y la generación de nuevas parcelas edificables. Esta alternativa se desarrollaría con el aporte de estudiantes y docentes de la CSU y la UPC.

De esta manera, comenzamos con dos ejercicios en paralelo. En primer lugar, propusimos a los profesores de CSU la presentación a los alumnos de un conjunto de casos de estudio de varios 
lugares el mundo para comprender el concepto de paisaje cultural y su aplicación en un proyecto concreto. La selección de casos responde por un lado a la complejidad del objeto de estudio, un sector de grandes dimensiones, con una geografía compleja, y por otra parte tiene que ver con historial de trabajos en el que viene participando la UPC y el Departamento de Urbanismo. Así, se presentaron los casos del Delta del Llobregat (Sabaté y Schuster, 2001), el Camino del Gaucho (Pesci y Pesci, 2000), el proyecto de la Quebrada de Humahuaca (Novick et. al., 2011), lugares asociados a eventos en Catalunya (Sabaté, Frenchamn y Schuster, 2004), el Parco Città en Italia y el caso de Xi Qiao, un desarrollo en torno a un volcán en Guandong.

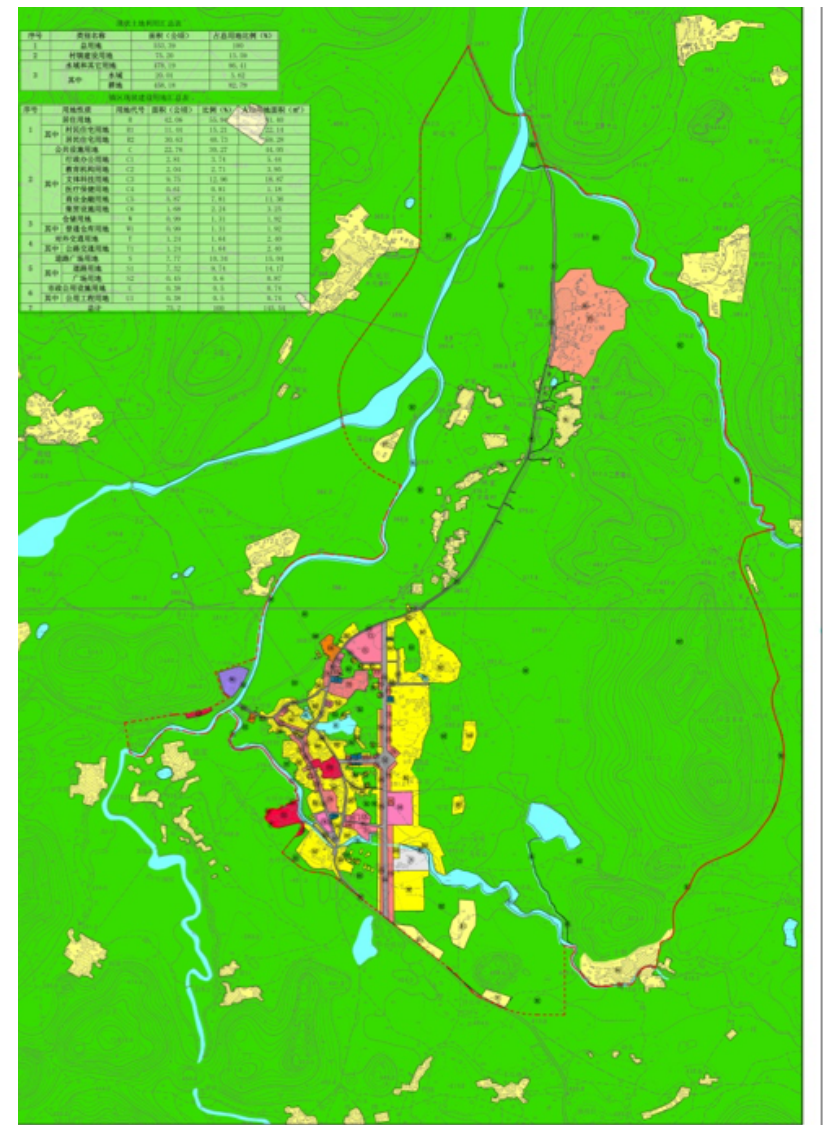

图例

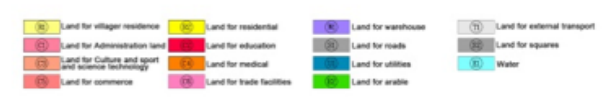

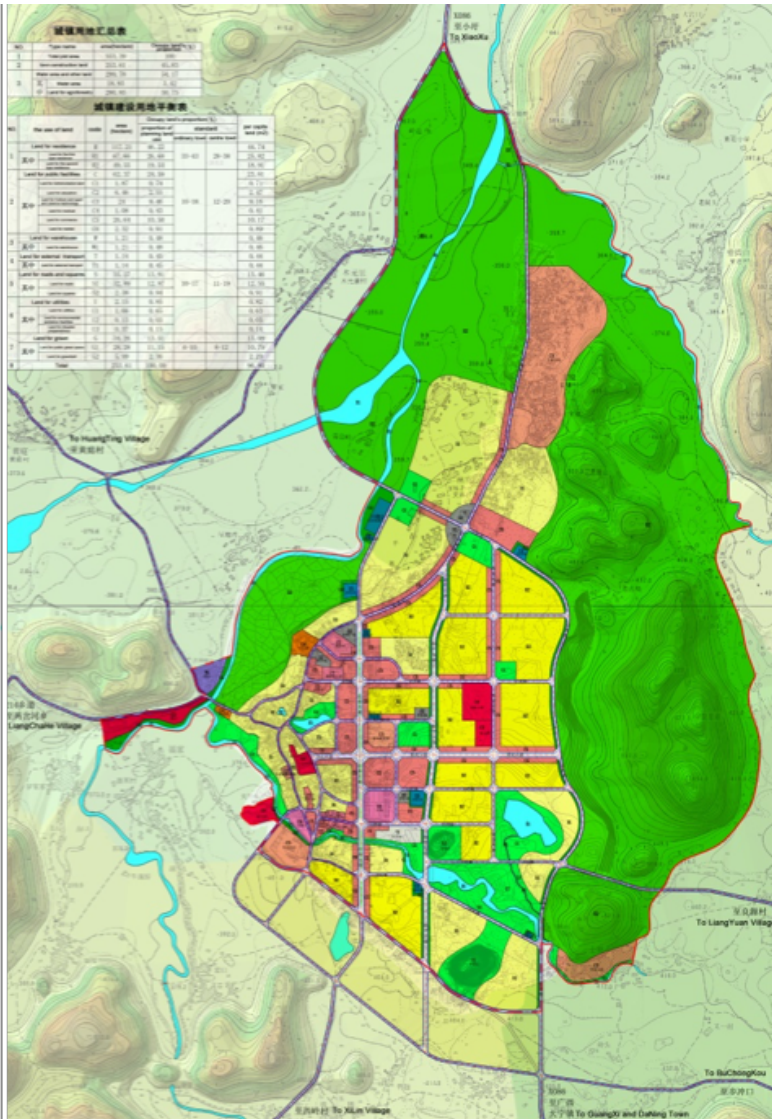

图例

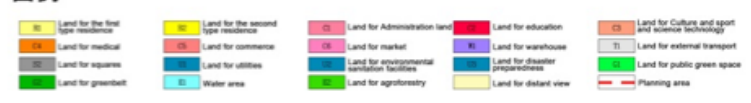

Figura 6. Daxu, situación actual y plan de crecimiento. Fuente: Daxu Municipality.

El segundo ejercicio consistió en un workshop de 10 días con la participación de docentes de ambas universidades, que incluía la visita al lugar. Durante esta visita, se elaboró un registro de las alternativas y posibilidades de Daxu, junto con registros minuciosos de los patrones urbanísticos, la morfología de la ciudad, su arquitectura, los modos de vida de la población, entre otros. Con este material, los estudiantes de máster más avanzados, comenzaron a plantear estrategias generales a nivel urbano y territorial, mientras que los alumnos de grado, empezaron a planificar proyectos específicos en diferentes áreas de la ciudad.

Al mismo tiempo, desde la UPC, se comenzó a trabajar en construir algunas lecturas interpretativas del territorio (en términos de Díaz Terreno, 2013) que nos permitieran comprender su conformación y transformación. Una primera lectura se centró en buscar la estructura primigenia de la ciudad, mediante la elaboración de una hipótesis de cómo sería la estructura urbana antes de la construcción del eje rectilíneo que hoy en día predomina sobre el resto (Figura 7). De esta manera, mediante el redibujo sobre el fotoplano de las construcciones más antiguas y su distribución, pudimos identificar dos rasgos centrales de la morfología urbana de Daxu. El primer rasgo que salta a la vista es la construcción de un sistema de poblados en racimo, unidos por caminos más o 
menos importantes, que van uniendo caseríos con diferentes entidades. Por otra parte, Daxu en su almendra central, se constituye en base a una estructura lineal que sirve para conectar otros asentamientos, condición que está reforzada por su posición central en el valle. Inferimos que este hecho ha ayudado a conceptualizar un nuevo crecimiento en base a una avenida Norte-Sur, que en parte replica de una forma abstracta esta condición.
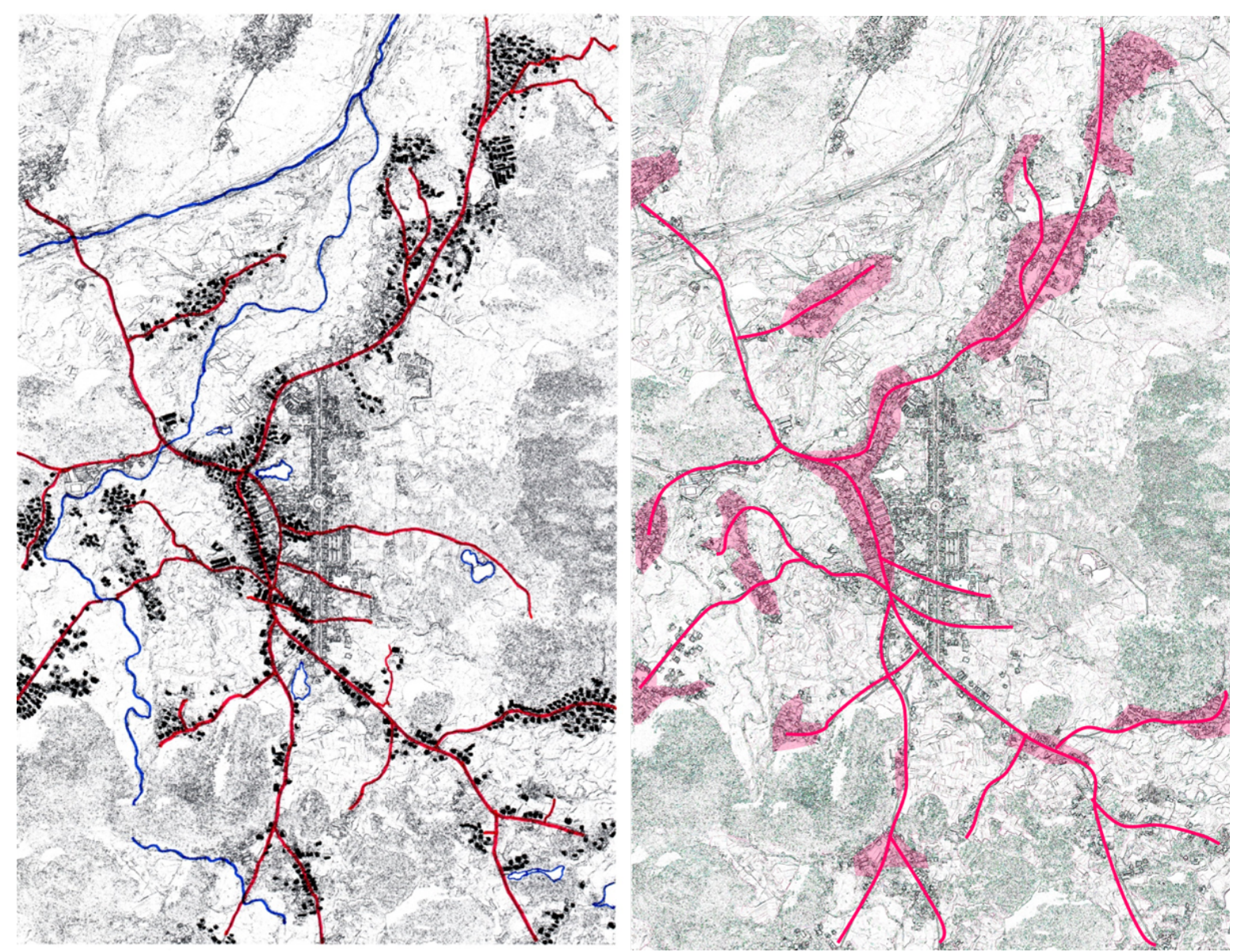

Figura 7. Hipótesis de construcción del asentamiento. Estructura en racimo sobre un eje Norte-Sur que pasa por el centro del casco antiguo de Daxu. Fuente: Elaboración propia.

También se desarrolló un estudio de la estructura de caminos (UPC), y un estudio de los usos del suelo (CSU). Juntos, permitieron pensar una estructura de recorridos paisajísticos, relacionando las tierras bajas cercanas al río y cultivos de arroz, con las vistas más cercanas a las montañas cársticas. Una especie de "anillo paisajístico".

La presencia del eje, por su parte, y la voluntad inamovible del municipio de plantear un crecimiento en malla, lleva pesar cómo se puede combinar un crecimiento en base a una geometría abstracta con un modo productivo y una estructura de pueblos más orgánica. Sobre la base de los caminos transversales, y el análisis de lo que ha dejado la combinación de la nueva arquitectura con la antigua, entendimos que era posible crear un nuevo tipo de "manzana", que denominamos híbrida (Figura 8). Una manzana de grandes dimensiones, con edificación perimetral, siguiendo el patrón deseado por el municipio, pero que en el interior conserva los trazados rurales y permite una edificación tradicional y una relación con el medio más cercana. De esta forma, se combinan dos patrones, de tradición y modernidad en una misma unidad urbana.

La construcción de estos bloques híbridos permite generar un crecimiento y una densificación de la ciudad, utilizando el sistema en malla, aunque readaptado, sin perder los modos de vida más tradicionales. Al mismo tiempo la preservación de estos corazones verdes en el interior de las manzanas híbridas, ayudaría a construir unos corredores verdes desarrollados en sentido Este-Oeste, a modo de dedos, que vinculan los sectores más naturales y las pequeñas montañas con la ciudad (Figura 10). 


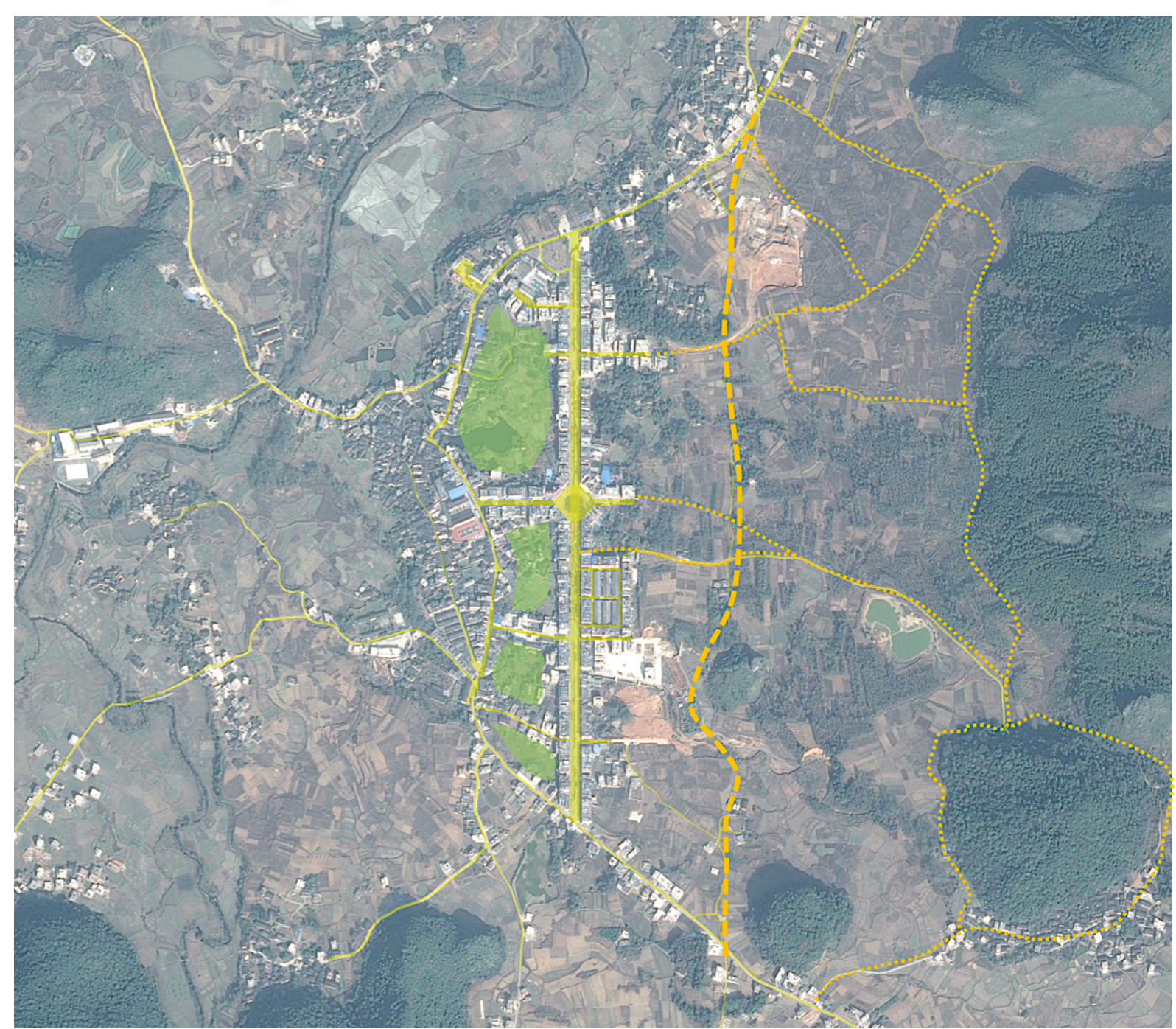

Figura 8. Estudio de los caminos que permitirían la construcción de un “anillo paisajístico”, y dinámicas de combinación de nuevas construcciones con la ciudad antigua. Fuente: Elaboración propia.

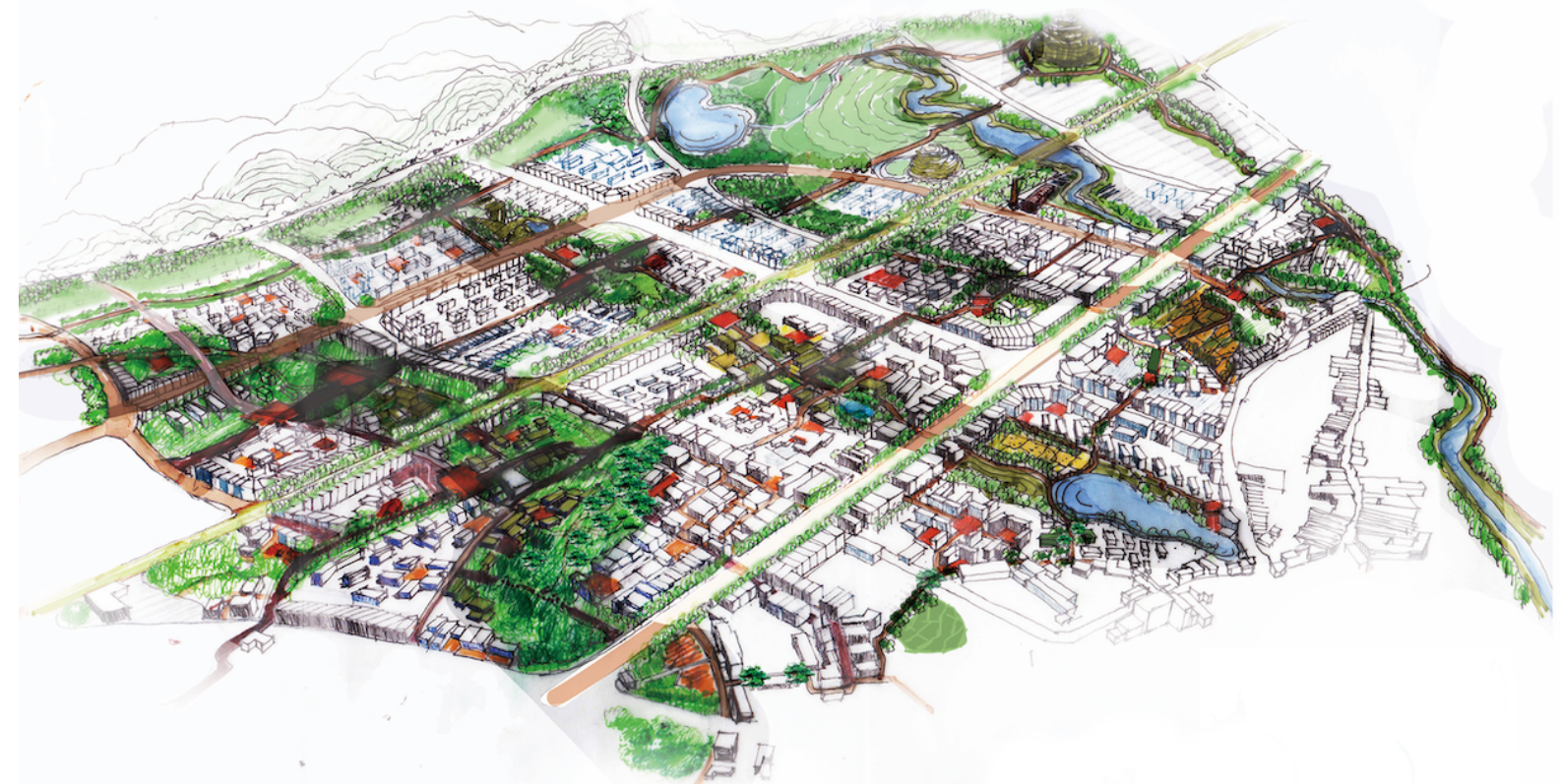

Figura 9. Imagen del futuro propuesto para la ciudad de Daxu, con los ejes verdes que conectan el poblado antiguo (en el extremo inferior derecho de la imagen) con la zona montañosa, en la esquina superior izquierda. Dibujo de los estudiantes: Meng Xiangru, Cao Fei, Zhang Wenbo. 


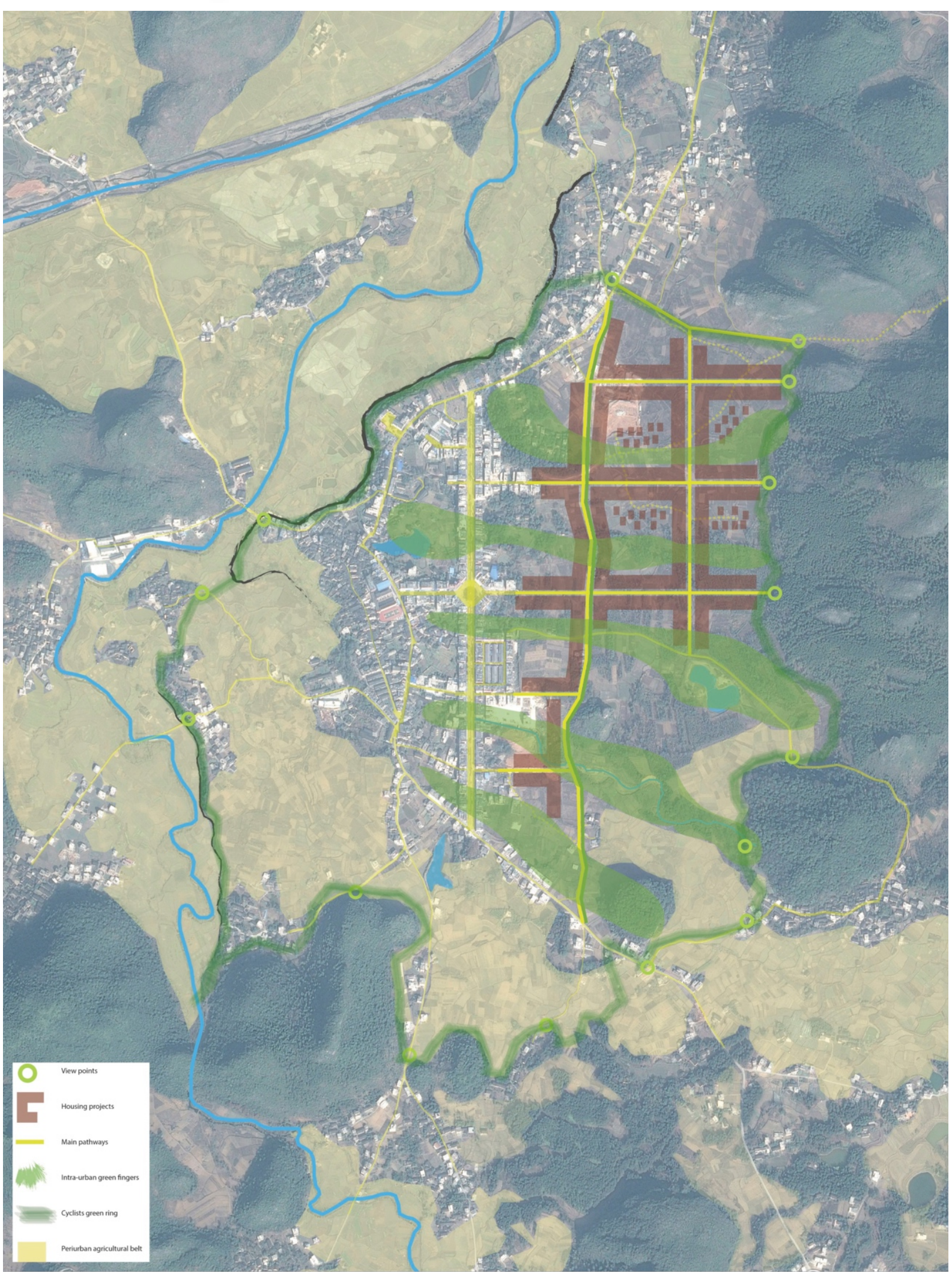

Figura 10. Dedos verdes que vinculan el casco antiguo de Daxu, la nueva avenida y el sector natural al Este de forma transversal. Fuente: Elaboración propia.

Un nuevo eje Norte Sur, que se desarrolla sobre un camino ya existente, pretende ser un recorrido más natural en contraposición a la gran avenida rectilínea. Aunque edificado en sus bordes, busca convertirse en una vía verde, amplia, arbolada y con recorrido apto para bicicletas. Este eje también vincula de una forma más natural el pueblo histórico de Baojing, ubicado en el extremo Norte, con la salida Sur de Daxu hacia el Sur y el Este. 
Sobre esta idea, los grupos de 3 o 4 alumnos trabajaron para profundizar en las directrices generales del crecimiento urbano y en las piezas arquitectónicas que podrían catalizar las transformaciones, vinculadas a la revalorización del centro histórico, a la actividad agrícola, a la percepción e interpretación del paisaje.

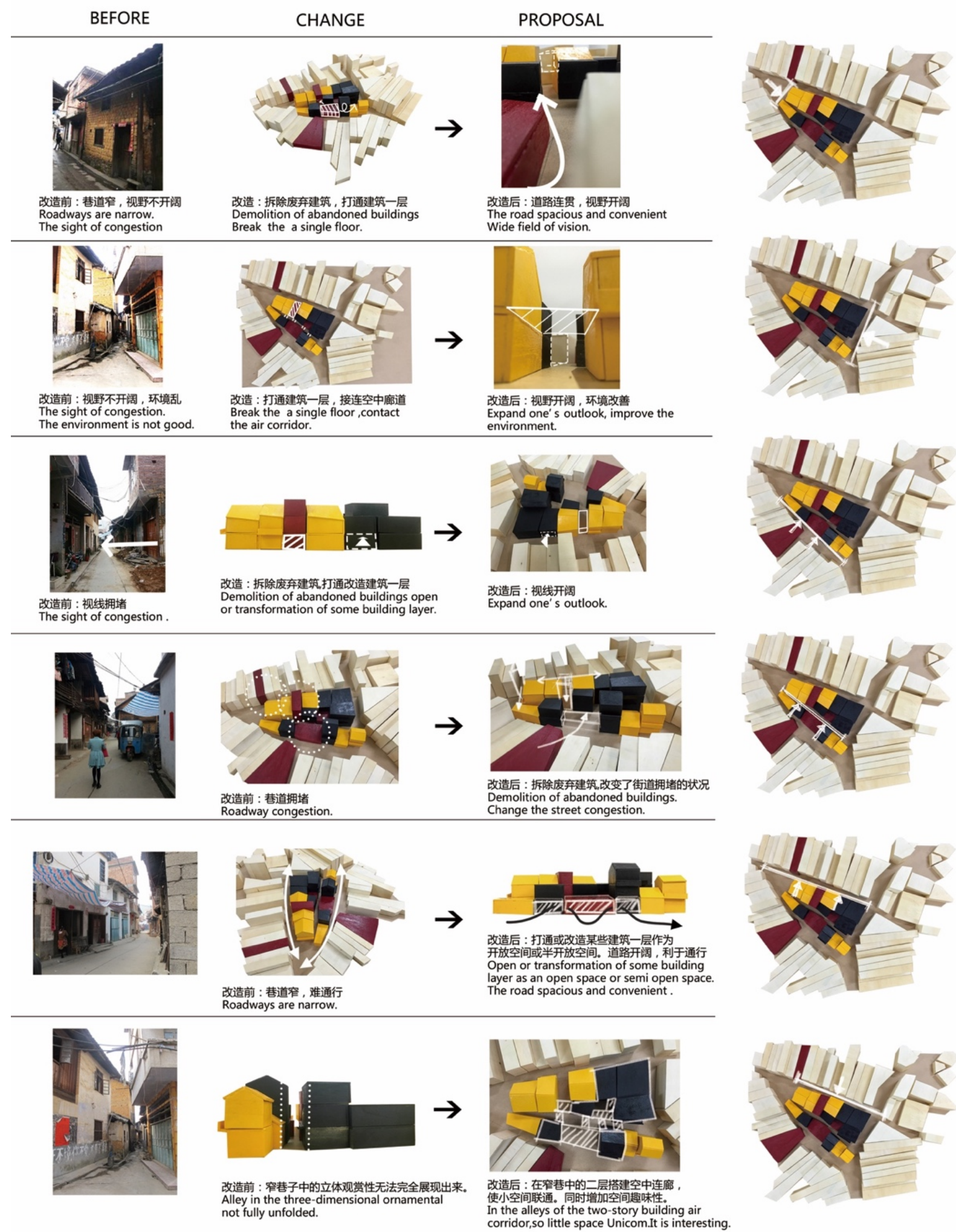

Figura 11. Propuesta para la revitalización del centro histórico. Fuente: Trabajo de los alumnos Qian Cong, Zhu Shuai, Ji Pinglan, Luo Xinyi. 


\section{Conclusiones: resultados y desafíos}

El contexto actual de acelerada urbanización en China propone nuevas problemáticas y desafíos. En cuanto a la conservación del patrimonio cultural y natural, el país asiático presenta múltiples diferencias de interpretación con respecto a occidente en general. Pero más allá de las diferencias, la experiencia de intercambio entre ambas universidades ha posibilitado un enriquecimiento mutuo en cuanto al proyecto de la ciudad y su territorio.

La utilización del concepto de paisaje cultural como guía o herramienta de proyecto ha permitido pensar de forma diferente las dificultades propias de la ciudad de Daxu, tales como el aislamiento con respecto al resto de la provincia, la escala de la ciudad en relación a su entorno o las problemáticas de índole económico. El ejercicio ha permitido mirar los activos de la región como valores especiales que pueden ser los protagonistas de un proyecto de crecimiento de la ciudad y de desarrollo local.

Creemos que estos valores deben ser entendidos por la población como tales, en un trabajo que implicará mucha mayor relación con la gente y quizá pueda ser el germen de una experiencia en la que ésta participe para entender el propio lugar en que vive. Este hecho ayudaría a reforzar algo que creemos es de suma importancia para llevar a cabo un proyecto como éste, que es la autoestima de los pobladores. Hemos comprobado a lo largo de las múltiples experiencias analizadas en distintos contextos que los proyectos más exitosos son aquellos en donde la población se encuentra activamente implicada.

Este planteo ha permitido dar una vuelta de rosca a la propuesta de la administración, que en un principio parecía inamovible, pero que esperamos que pueda retomar algunos de los aspectos trabajados en pos de incorporar más variables a la ordenación urbanística planteada, más allá de la atención sólo al trazado.

\section{Referencias}

Bottazi, J., Maire, R., Vanara, N., Bruxelles, L., \& Barbary, J.(2011, enero). Le patrimoine karstique de la Chine du Sud-Ouest : contexte géotectonique, genèse du karst et rôle de l'effet de site. En: Les Cahiers d'Outre-Mer, no 253-254, pp. 151-168. En línea: http://com.revues.org/6211 ; DOI : 10.4000/com.6211

Conti, A. (2012). Paisajes culturales: la interacción entre el hombre y la naturaleza. Trabajo sin publicar, disponible en: https://www.academia.edu/5833242/Paisajes_culturales

Corboz, A. (2001). El territorio como palimpsesto. En: A.M. Ramos (2004). Lo urbano en 20 autores contemporáneos. Barcelona: Edicions UPC.

China Institute (2011). http://www.china-institute.org/articles/Les_minorites_en_Chine.pdf

Díaz Terreno, F. (2013). Constelaciones rurales serranas: lógicas de ocupación del territorio y modelos de orden en el Norte de Traslasierra, Córdoba, Argentina. Tesis doctoral leída en la Universidad Politécnica de Cataluña, Barcelona, España.

Novick, A. (Dir.), Favelukes, G. (Coord.), Catenazzi, A., Cañelas, E., Mancini, C., Tommei, C., Potocko, A., \& Vecslir, L. (2011). Las transformaciones del territorio. Mapas, patrimonio y lugares de la Quebrada de Humahuaca. En: Seminarios de crítica no. 169. Buenos Aires: IAA.

Pesci, R., \& Pesci, L. (2000). "Camino del Gaucho. Paisaje Cultural y Desarrollo sustentable, un camino de oportunidades y cooperación para el Mercosur". En: R. Pesci. Del Titanic al Velero. La Plata: Fundación Cepa.

Sabaté, J. (2002). “En la identidad del territorio está su alternativa”. En: Revista Ingeniería y Territorio, $n^{o}$ 60, pp. 12-19. Barcelona.

Sabaté, J. (2015). Entrevista a Joaquín Sabaté, sobre el libro Parques Mineros, Ecomuseos y Geoparques: Estrategias de puesta en valor / Editorial Stoq. Noviembre 2015. En línea: <http:// 
www.plataformaarquitectura.cl/cl/776032/la-preservacion-a-traves-de-la-transformacion-paisajesculturales-de-la-mineria-por-joaquin-sabate-bel-en-parques-mineros-stoq>

Sabaté, J., \& Galindo, J. (2009). “El valor estructurante del patrimonio en la transformación del territorio". En: Apuntes 22(1), pp.20-33. Bogotá, enero-junio.

Sabaté, J., \& Schuster, M. (2001). Projectant l'eix del Llobregat: Paisatge cultural i desenvolupament regional / Designing the Lobregat Corridor: Cultural Landscape and Regional Developement. Barcelona: UPC/MIT.

Sabaté, J., Frenchman, J., \& Schuster, M. (2004). Llocs amb esdeveniments / Event places. Barcelona: UPC/MIT.

Sauer, C. (1925). "The morphology of landscape". En: J. Leighly (ed.). Land and life: a selection from the writings of Carl Ortwin Sauer. Berkeley: University of California Press, 1963 [1925].

Solá-Morales, M. de (coord.) (1981). “La identitat del territori català". En: Revista Quaderns d'arquitectura i urbanisme, Vol. Extra., COAC, Barcelona.

Wagner, P., \& Mikesell, M. (ed.) (1962). Readings in Cultural Geography. Chicago: The University of Chicago Press.

Wiens, H. (1967). Han Chinese expansion in South China [archive]. Shoe String Press. p. 276. 